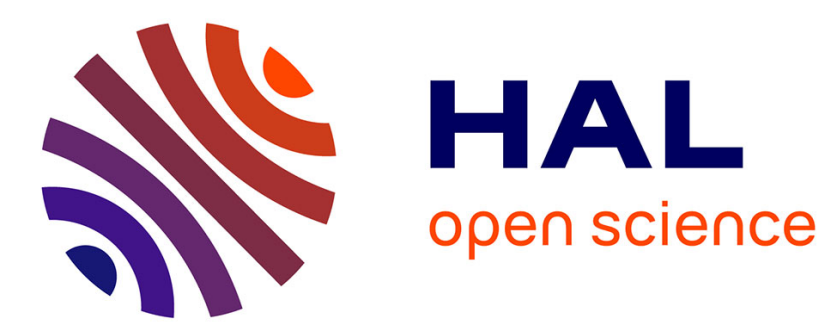

\title{
L'inscription du vatt Mahā Lābh K. 1046
}

Olivier de Bernon

\section{- To cite this version:}

Olivier de Bernon. L'inscription du vatt Mahā Lābh K. 1046. Bulletin de l'Ecole française d'ExtrêmeOrient, 2017, 103 (1), pp.433 - 452. 10.3406/befeo.2017.6259 . halshs-02537863

\section{HAL Id: halshs-02537863 \\ https://shs.hal.science/halshs-02537863}

Submitted on 9 Apr 2020

HAL is a multi-disciplinary open access archive for the deposit and dissemination of scientific research documents, whether they are published or not. The documents may come from teaching and research institutions in France or abroad, or from public or private research centers.
L'archive ouverte pluridisciplinaire HAL, est destinée au dépôt et à la diffusion de documents scientifiques de niveau recherche, publiés ou non, émanant des établissements d'enseignement et de recherche français ou étrangers, des laboratoires publics ou privés. 


\section{L'inscription du vatt Mahā Lābh K. 1046}

\section{Olivier de Bernon}

\section{Citer ce document / Cite this document :}

de Bernon Olivier. L'inscription du vatt Mahā Lābh K. 1046. In: Bulletin de l'Ecole française d'Extrême-Orient. Tome 103, 2017. pp. 433-452;

doi : https://doi.org/10.3406/befeo.2017.6259

https://www.persee.fr/doc/befeo_0336-1519_2017_num_103_1_6259

Fichier pdf généré le 08/11/2019 


\title{
L'inscription du vatt Mahā Lābh K. 1046
}

\author{
Olivier DE BERNON*
}

Mots-clés : Cambodge; province de Tpūn Ghmuṃ; inscription; règne de Sisowath; rituel de pose des sìmā.

Keywords: Cambodia; province of Tpūn Ghmuṃ; inscription; reign of Sisowath; ritual of laying simm $\overline{\text {. }}$

L'inscription K. 1046, détruite entre 1975 et 1979', nous est connue grâce aux photographies qu'en avait fort heureusement faites en 1962 Claude Guioneau (1925-2017)² pour son ami Claude Jacques (1929-2018), alors membre de l'EFEO, grand spécialiste de l'épigraphie sanskrite du pays khmer. C'est à la mémoire de ces deux amis et connaisseurs exceptionnels du Cambodge que cet article est dédié.

\section{Origine de l'inscription}

L'inscription K. 1046 se trouvait autrefois au pied de l'autel du Buddha à l'intérieur du vihāra du vatt Mahā Lābh ${ }^{3}$ (/moha: liəp/), situé sur la berge orientale du Danle-tūc («le petit fleuve»), faux défluent du Mékong qui s'écarte du cours majeur du fleuve vers l'est à la hauteur de Kompong Cham et le rejoint en aval des Quatre-Bras, dans le nord de la province de Prey Veng.

Les textes qui la composaient étaient gravés sur chacune des faces d'une stèle plate d'environ un mètre de haut dont le sommet mouluré en forme d'accolade était orné de motifs floraux. Ils étaient inscrits en grands caractères $m \bar{u} l$ modernes de plus d'un centimètre de haut, retracés à la peinture blanche sur un méplat recouvert de laque noire auquel un épais rebord doré servait de cadre. Le bas de la stèle, formant tenon, s'encastrait dans un socle rectangulaire orné de pétales de lotus stylisés qui seul subsiste en place.

L'inscription indique (face 2, lignes 6, 22, 24) que le vatt Mahā Lābh se trouvait dans la «province» (khett) de Tpūn Ghmum («la tête de l'abeille»).

\footnotetext{
* École française d'Extrême-Orient, olivier.de-bernon@efeo.net.

1. D'après de nombreux témoignages recueillis sur place, le 10 septembre 1999.

2. Claude Guioneau fut, à la fin des années 1950 et jusqu'en 1964, pilote de l'avion des plantations de Snuol. Photographe reconnu, on lui doit un grand nombre de photos publiées ensuite, sans toujours mentionner son nom, par Bernard Philippe Groslier, ainsi que plusieurs clichés utilisés par le ministère de l'Information du Gouvernement royal, notamment ceux, très précieux, des splendides peintures murales du vihära de la Pagode d'argent avant leur destruction inconsidérée lors de la rénovation du bâtiment en 1966 (ministère de l'Information 1962: 260).

3. Le parti est pris ici, ainsi que dans la traduction et les remarques qui la suivent, de transcrire tous les mots avec une majuscule dès lors qu'il s'agit d'un nom propre de lieu ou de personne.
} 
Cette «province», ravalée temporairement au statut de «district» (sruk), s'est trouvée incluse à compter de 1892 dans la «Résidence», devenue par la suite «province», de Kompong Cham. Elle a été recréée en 2013 par la scission d'une partie de la province de Kompong Cham mais avec un contour différent de celui qui était le sien au XIX ${ }^{\mathrm{e}}$ siècle. La limite occidentale de la nouvelle province de Tpūn Ghmum ne passe plus comme autrefois le long du Danle-tūc mais un peu à l'est, le long des berges des deux vastes marais que sont le pin Krabī et le piñ Tren. Ainsi le vatt Mahā Lābh se retrouve-t-il désormais dans le district de Koh Sūdin de la province de Kompong Cham.

Dans le Cambodge à la période moyenne, la province de Tpūn Ghmum, lorsque son tracé incluait le vatt Mahā Lābh, avait une importance symbolique considérable quoique assez obscure. Elle faisait partie, en effet - avec les khett Kaṃbañ Svāy ou Sandak (dans l'actuelle province de Kompong Thom), Drāṃn (dans l'actuelle province de Takéo), Pā Bhnaṃ (dans l'actuelle province de Prey Veng) et Bodhisāt' - des cinq «territoires» (țī) placés rituellement sous l'autorité de l'un des cinq «rois feudataires» (stec trāñ ') qu'étaient l'Uk-ñā Dhammātejo, l'Uk-ñā Tejo, l'Uk-ñā Bisṇulok, l'Uk-ñāAr-jūn et l'Uk-ñā Suorgālok (Leclère 1894: 183; Aymonier 1900: 70; Mak Phœun 1995: 139, n. 161).

Progressivement, à compter du milieu du XVIII ${ }^{\mathrm{e}}$ siècle, la province de Tpūn Ghmum s'est trouvée majoritairement peuplée de Chams, au point qu'ils tentèrent d'y créer un état indépendant (De Féo 2005 : 102). En 1858, après avoir conduit lui-même une expédition militaire, le roi Ang Duong déporta ces populations dans le reste du pays avant d'entreprendre de restaurer le lien spécifique de ce $t \grave{l}$ avec sa couronne. Quarante ans après la fin des violences qui avaient marqué cette province, la construction ou la reconstruction du vatt Mahā Lābh sous le patronage royal procédait encore de l'entreprise de réappropriation de ce territoire par les Khmers.

\section{Réputation du vatt Mahā Lābh}

Le nom du vatt Mahā Lābh pourrait signifier littéralement «monastère de la grande fortune». Une tradition locale pose cependant qu'il dérive du surnom de son fondateur, un «moine lettré» (mahā), venu lui-même du village de Brèk Lābh («l'arroyo de la bonne fortune») qui s'enfonçait, en face de Phnom Penh, dans la berge du Tonlé Sap. Il s'agit donc du «monastère du vénérable [venu] de Brèk Lābh.»

George Groslier a célébré l'exceptionnelle beauté du vatt Mahā Lābh (Groslier 1931 : 28). Bien que passablement déjetés dans les années 1990 , les bâtiments de ce monastère offraient encore l'un des plus beaux exemples de l'architecture religieuse khmère de la seconde moitié du XIX ${ }^{e}$ siècle et du début du $\mathrm{xx}^{\mathrm{e}}$ siècle. Ils formaient un vaste ensemble de locaux d'habitation et d'enseignement en maçonnerie - malheureusement remplacés récemment par des constructions de moindre allure -, au centre desquels se dressaient plusieurs bâtiments de bois qui, par chance, subsistent encore. Le plus remarquable de ces édifices de bois est le «sanctuaire» (vihāra) dont les structures portantes, les piliers et les pièces maîtresses de la charpente, sont 
entièrement couvertes de «laque noire» $\left(m r a \bar{a} k^{\prime}\right)$ ornée de dessins au pochoir à la feuille d'or (Le Bonheur 1989: 255, fig. 704; Nafilyan 1997: 13).

On peut voir également, à proximité du vihāra, une exceptionnelle «cellule de passage» (brah banlā) offerte par le roi Norodom (1835-[r.1860]1904) au Suprême Patriarche Nil Dīen (/nel tiong/) (1823-[s.p.1857]-1913) au tournant du $\mathrm{xx}^{\mathrm{e}}$ siècle. Le vatt Mahā Lābh était, en effet, l'un des monastères de villégiature de ce religieux prestigieux, personnage considérable dans l'histoire moderne du bouddhisme khmer, chef de la fraternité mahānikāy sous les règnes d'Ang Duong, de Norodom et de Sisowath.

La lecture de ces deux textes ne présente pas de difficulté particulière malgré l'abondance, parfois fastidieuse, de formules emphatiques stéréotypées utilisées dans l'enoncé des titres des principaux personnages et pour la désignation de certains actes monastiques.

\section{Le texte}

[Face I (fig. 1)]

1. sabbhamasatu varamanggalā jaiyyāratirek braḥ buddh-sakrāc braḥ sāsnār pān 2437 braḥ vasār

2. mahā sakkharāc pān 1816 brah vassār . cal-sakkharāc pān 1256 brạ̣ vassār . lok $^{\circ}$ grūv

3. braḥ cov aț̣hikā vatth mahā lāb braḥ nām Ind Jot jā braḥ Upajjhā . kāl git sān brạ̣ vîhā neh

4. kālnoḥ braḥ gun braḥ ariyyasatthā nin braḥ gun braḥ ariyyaghosār jā grūv ātmā dāṃn ${ }^{\circ}$ bìy ${ }^{\circ}$ brah an gu gun vatth

5. kantāl koḥ suddin höy ${ }^{\circ}$ dran̉ bīcāraṇā sāpāy ${ }^{\circ}$ knan brah rājj-hāradaiy ${ }^{\circ}$ dran̉ mān braḥ buddh-țîkā pangāp

6. prör thā Aoy ātmār-bhābv dhvör bra vīhā neh ddov tpit chānd kānd tè cās ṇậ höy ${ }^{\circ}$ dān chand nov nin juoy

7. daṃnuk aṃrun rīep-caṃ Aoy ${ }^{\circ}$ dhvör ātmā ka daduol tām brạ̣ buddhțịkār höyo pān mak git nin lok bhikkhu jā

8. sös ka bram daduol tām grubv an̉g git dov rak jhö grön lör khluon en pān grubv nāṃm mak țal

9. noḥ as pāsak ñāt ñom anak muk anak kā Aok-ñā bañā brah sruk jit sruk chñāy ${ }^{\circ}$ sāpāy ${ }^{\circ}$ sā-

10. dhdhār Ḷ̂ thā ātmār git sān braḥ vīhā noḥ kă nāṃm gnār git pān pracaiyo yak mak pragen $\mathrm{Aoy}^{\circ}$ diñ

11. sasar neḥ pān grupv saṃpp dāṃn ${ }^{\circ} a^{\circ}$ tamlaiy $^{\circ}$ jā prāk 20 ṇèn dhvör braḥ vīhār neh sut

4. Photo de l'intérieur du vatt Mahā Lābh par George Groslier en 1929. 
12. tè lok bhikkhu jā sös dhvör dāṃn ${ }^{\circ}$ as gmān jhnuol der kāl tèl git lök noḥ brạ gun

13. braḥ nām braḥ arīyyasatthā jā mcāḥ lok dran̉ braḥ ābāt jā duṃmn்un as brah jun dauv nauv tè

14. braḥ gun braḥ nām braḥ ariyyagosā braḥ ang noḥ brạ̣ cov atthikār ātmār pān dov

15. nīmant lok mak jā mañgal ddhạ̣ prajuṃm kār e lok āccāray noḥ pān añjöñ lok grūv

16. dāṃn ${ }^{\circ}$ bīy ${ }^{\circ}$ braḥ nām jhmoḥ lok āccārīy an 1 āccārīy kèr 1 nin āccārīy an jā anak amm-

17. run rīep blīy ${ }^{\circ}$ ār prakās añjöñ devațār mak jā pathaṃ kā ṛk pān bīlā lök brạ̣ vih̄ā

18. neh nov kāl paccapan thniaiy ${ }^{\circ}$ ccānd 1 roj khè phalgun chnāṃ mamī chasaks srec papuor jā mangal țạ̣-

19. kal duk knun̉ braḥ sāsnār tă rīen dov lök braḥ vīhā neḥ knun chnāṃ mamī dhvör rīen tă mak

20. țal chnāṃ thoh yak jān mak gūv phtèr pit māso sasar ruoc srec knun chnām ron chasaks

21. braḥ sāsnār pān 2447 braḥ vassār rī phallā"-annisañg tèl sān brah vîhā neh

22. ātmār sūm thvāyo braḥ rājjakusal Aoy ${ }^{\circ}$ pān țal dọ̣ov braḥ gun jā mcāḥ dāmin ${ }^{\circ}$ bìy ${ }^{\circ}$ braḥ añg nau gun

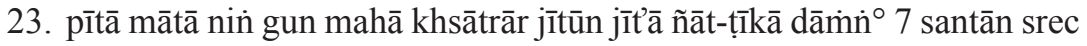
papūr țam- ${ }^{5}$

24. tammkal duk knuñ sāsnār brạ̣ gun brạ̣ nām brạ̣ samannagotam jā mcāḥ Aoy ${ }^{\circ}$ run-rön tamkal Aoy

25. țal brah si-ār pān trāḥ srec papūr . / . braḥ cov attīkā ātmābhābv tāio ${ }^{\circ}$ akkhaŕā

26. jā saṃnūm duk $A_{0 y}{ }^{\circ}$ ñom dāṃn ${ }^{\circ}$ as gnār tẹl mān cit sādhdhā pańköt knun asañkhārik rălk tal

27. braḥ gan dān ${ }^{\circ}$ as höy ${ }^{\circ}$ pān yak pracaiy mak pragen ātmār das ppī tic crön sin pān grubv gnār

28. cūl sān braḥ vîhā neh Iḷūvneh ātmār sān rūc höy ${ }^{\circ}$ ṭucneh ka sūm Aoy ${ }^{\circ}$ pān kusal dāṃn ${ }^{\circ}$ ampāâl ṇeḥ

29. juoy jūn $A_{0 y}^{\circ}$ pān saṃrec măg phal țal Upāa sak $\left.\begin{array}{l}\text { sak } \\ \text { sikā }\end{array}\right\}$ țal brạ̣ si-āa pān trās ${ }^{\circ}$ kālnār sūm $A_{0 y}^{\circ}$ pān

30. saṃrec mag phal țal braḥ nībān jā sṭhān parummm-suk prasör srec papūr han. /.

5. La syllabe tam- inscrite à la fin de cette ligne est superflue car le mot complet $[$ tammkal $<$ tam$\left.\mathrm{kal}^{\prime}\right]$ est repris au début de la ligne suivante. 


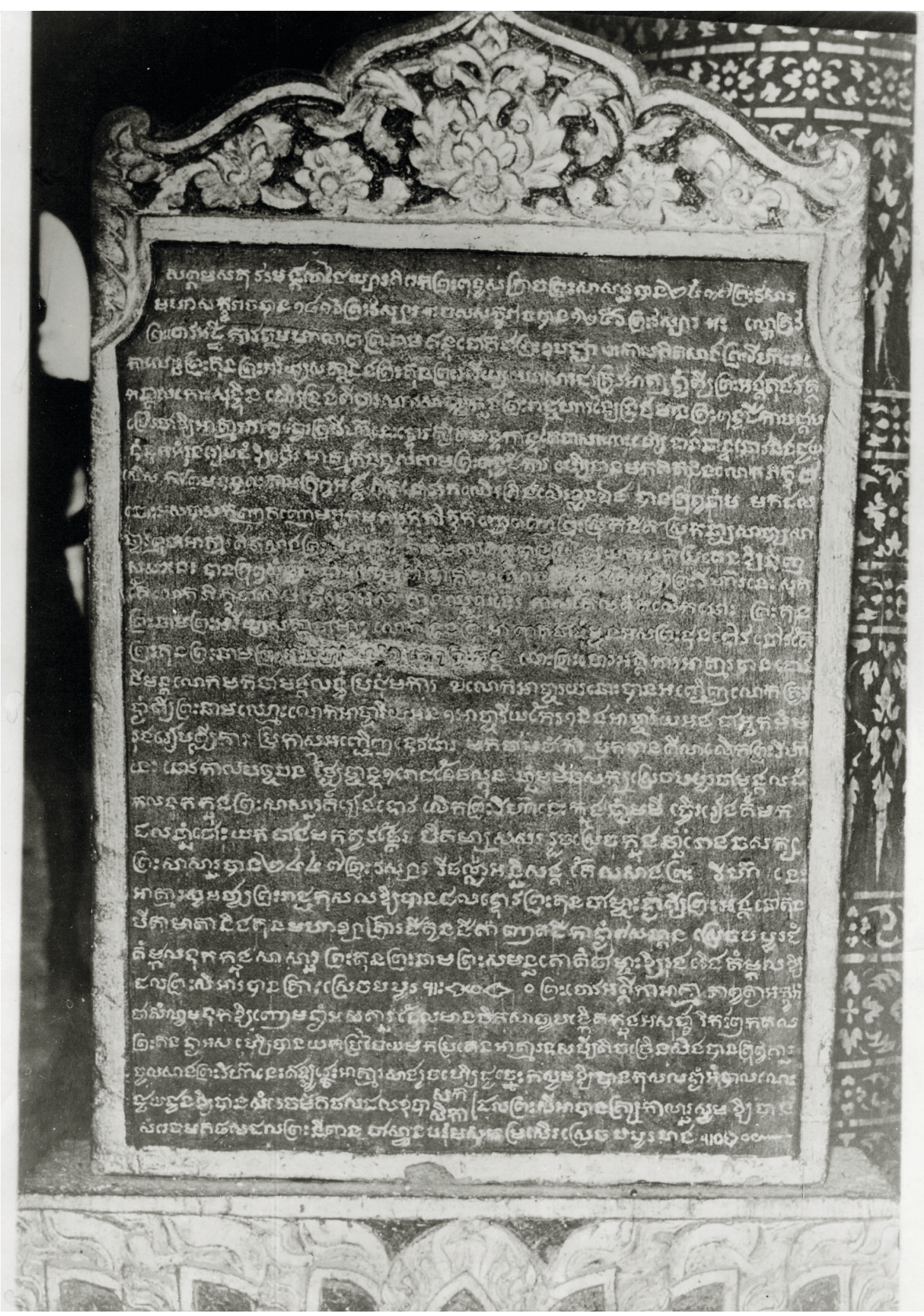

Fig. 1 - Inscription du vatt Mahā Lābh K. 1046, face I. (Cliché: Claude Guioneau, 1962.) 


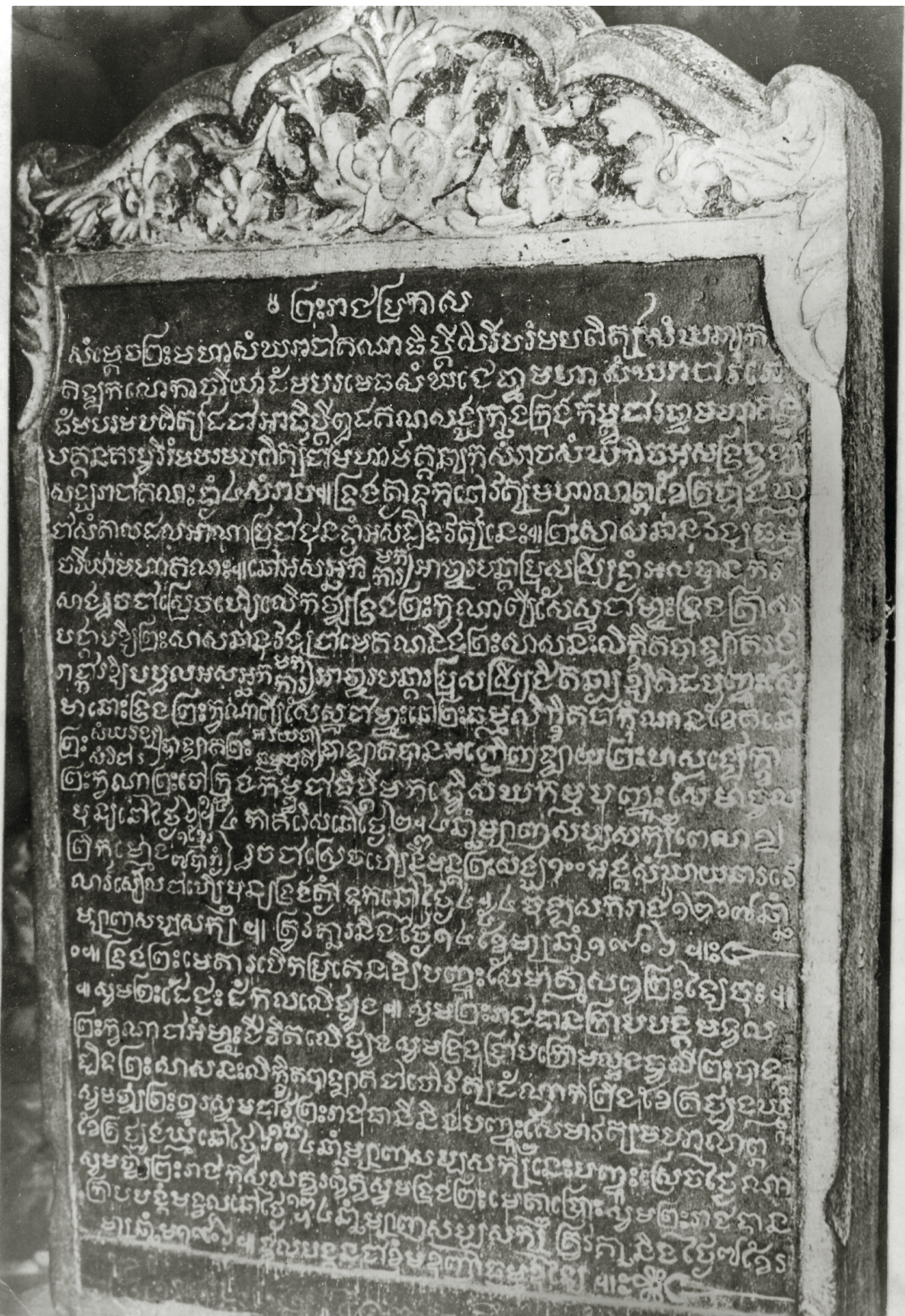

Fig. 2 - Inscription du vatt Mahā Lābh K. 1046, face II. (Cliché: Claude Guioneau, 1962.) 
[Face II (fig. 2)]

1. braḥ rāj-prakās

2. saṃmtec braḥ mahā saṃgharājā gaṇādhiptī sirī paraṃm pabity saṃgha nāya ${ }^{\circ} \mathrm{k}$

3. tiḷkkalokācarīyā țammm paramedh saṃgh jedhṭhā mahā saṃgharājā varo-

4. țạnm param pabity ța jā ādhiptī aiṭa gaṇasañgh knuñ kruñ kambūjāradhdhā mahā inda-

5. patt nagar pūrīraṃm param pabity jā mahā magg nāy ${ }^{\circ}$ ak saṃrāc saṃghakic as dradūnis

6. sanghh rājāgaṇaḥ dāṃn 4 saṃrāp . dran̉ tān ${ }^{\circ}$ duk nau vats mahā lābbh khètr thpūn ghmạ̣

7. jā saṃāl țal āṇāprajājun dāṃn ${ }^{\circ}$ as ț̣id vats neh . brạ̣ sāsanānuvañs dhamma-

8. carīyā mahā gaṇaḥ . nau as anak $\left.\begin{array}{c}\text { mak } \\ \text { kār }\end{array}\right\}$ āccār pantā prus srīy dāṃn ${ }^{\circ}$ as pān kar-

9. sān ruoc jā srec höy ${ }^{\circ}$ lök thvāyo dran braḥ kūṇā bīy ${ }^{\circ}$ sèsṭh jā mcāḥ dran trās

10. pangāp Aoy $^{\circ}$ brah sāsanā'nuvañs jā megaṇ nin braḥ sāsanaḥ likkhit pāṭāt ran

11. rājkār Aoy papuol as anak $\left.\begin{array}{c}\text { mak } \\ \text { kār }\end{array}\right\}$ āccār pantār prus srīy ${ }^{\circ}$ jit chñāy ${ }^{\circ}$ Aoy ${ }^{\circ}$ git pañcuh sè-

12. mā noḥ dran̉ braḥ kūṇā bīy ${ }^{\circ}$ sèsṭh jā mcāḥ nau braḥ dhammalikkhit jā kuṃnān khèt nau

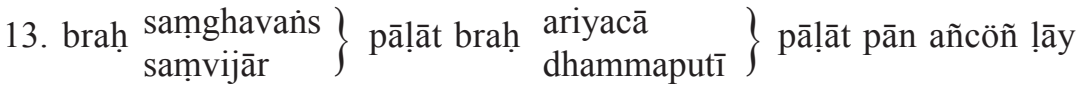
brạ̣ hasaḷkkhā

14. braḥ kūṇā braḥ cau krun kambūjādhiptī mak dhvö saṃghakamm pañcụ̣ sèmā cūl

15. puny nau thñai ${ }_{6}^{15} \cdot 4$ kāt rös nau thñai $2 \cdot 3$ chnāṃ msāñ sappasaks belā $E$

16. brik mmon் $\left.\begin{array}{l}1 \text { khmèr } \\ 7 \text { pārāminn }\end{array}\right\}$ ruoc jā srec hoÿ nīmant braḥ sangh 100 ang saṃghāyanār ve-

17. lā răsīel jā höy ${ }^{\circ}$ puny drañ tāṃn ${ }^{\circ}$ duk nau thniai $4 \cdot 4$ cuḷasakarāj 1267 chnāṃ

18. msāñ sappasaks . trūv gnār nin thniai 14 khè mās ${ }^{\circ}$ chnāṃ1906 . /

19. dran braḥ metār pök pragen $A_{0 y}^{\circ}$ pañcuh sèmā tāmº sabv braḥ daiy ${ }^{\circ}$ cuh .

20. sūm braḥ țèjjah țạ̣kal lö thpūn . sūm braḥ rājadān krāp pangaṃm dūl

21. braḥ kūṇā jā aṃmcāḥ jīvit lö thpūn sūm drañ jrāp krom l-an̉ dhūlī braḥ pād

22. țpid brah sāsanah likkhit pāḷât jā cau vats țạ̣nāk brīn khètr thpūn ghmum 
23. sūm thvāyo braḥ bvar sūm jāv braḥ rājadhānī nin pañcuh sèmā vats ${ }^{\circ}$ mahā lābbh

24. khètr thpūñ ghmaṃ nau thñai $6 \cdot 4$ chnāṃ msāñ sappasaks neḥ pañcuh srec thniai ñā

25. sūm thvāy ${ }^{\circ}$ brah rajakusal guor bvaṃ guo sūm dran braḥ metā proḥ sūm brạ rājadān

26. krāp paṅgaṃm dūl nau thniai $\stackrel{13}{\cdot} \cdot 4$ chnāṃ msāñ sappasaks trūv gnā nin thñai 7 khèr

27. mās ${ }^{\circ}$ chnāṃm 1906 . dūl pañgan jā khñaṃm Uñā dhamma Uvdaiy $^{\circ}$.

\section{Traduction}

\section{[Face I]}

Puissiez-vous recevoir le succès, la chance la plus grande et la victoire la plus extrême ${ }^{6}$ !

L'ère bouddhique ayant duré 2437 années, (2) la grande ère ayant duré 1816 années, la petite ère ayant duré 1256 années, le (3) chef du monastère de Mahā Lābh, du nom de Inda-Joti, ayant rang de précepteur [pour les ordinations], a eu l'idée de construire ce sanctuaire ${ }^{7}$.

(4) À cette époque, le Braḥ Gun Braḥ Ariyasaddhā et le Braḥ Guṇ Ariyaghosa qui, tous deux, avaient été mes maîtres, résidaient au vatt (5) Kaṇtal, à Koh Sūdin. Ils examinèrent [cette idée], en eurent le cœur réjoui. Ils m'enjoignirent ${ }^{8}(6)$ de construire ce sanctuaire sans délai ${ }^{9}$, parce qu'ils ${ }^{10}$ se faisaient très vieux déjà, [mais] que, tant qu'ils demeuraient vivants, ils étaient disposés (7) à m'aider ${ }^{11}$ dans cette tâche. Ayant entendu ces paroles, je me suis concerté ${ }^{12}$ avec [tous] les religieux qui avaient été (8) [leurs] élèves, [afin] que chacun d'entre eux convienne de rechercher

6. Sabbhamasatu varamangalā jaiyyāratirek ( $<$ śubha astu varamañgalā jaya atireka): formule d'exorde stéréotypée, commune à partir du XVI ${ }^{\mathrm{e}}$ siècle (voir, par exemple, les inscriptions K. 805 [1535 A.D.], K. 75 [1628 A.D.], ainsi que la plupart des inscriptions modernes d'Angkor, de l'IMA 6 [1599 A.D.] à l'IMA 39 [1747 A.D.]).

7. Ce premier paragraphe ne contient pas de pronom réflexif, mais il faut comprendre que le personnage qui y est désigné est bien l'auteur de l'inscription, le même qui utilise, par la suite, le pronom a $\bar{t} t m \bar{a}$ ou $\bar{a} t m \bar{a} r$-bhābv (ligne 6) (< a tmābhāa) équivalent au pronom de la première personne pour un religieux. Le titre de ce religieux - brah sāsanānuvañs dhammacarīyā mahā gana: - est donné dans l'inscription de la face II, lignes 7 et 8 .

8. Mot à mot: mān brah buddh-țìka «dire [pour un buddha ou un religieux]»+ pañgāp

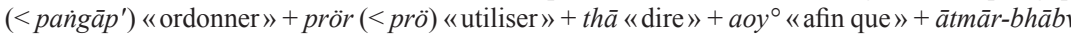
$(<\bar{a} t m \bar{a} b h \bar{a} p) \ll \mathrm{je} »+$ dhvör $(<d h v \ddot{o}) \ll$ faire».

9. Cette proposition ne comporte pas d'expression temporelle, mais elle se termine par l'explétif ddov (dau) «aller», ayant ici valeur d'impératif atténué.

10. chānd est un pronom archaïque, utilisé par un vieux moine s'adressant à un plus jeune. Normalement utilisé au singulier, il semble pourtant se rapporter ici aux deux maîtres de l'auteur que sont le Braḥ Guṇ Braḥ Ariyasaddhā et le Braḥ Guṇ Ariyaghosa.

11. Mot à mot: juoy «aider» + damnnuk «méthode» + aṃrun் (< baṃruñ) «être prêt».

12. Mot à mot: mak «venir»+ git «penser». 
du bois de charpente par ses propres moyens ${ }^{13}$ et que, quand il en aurait suffisamment, il l'achemine ${ }^{14}$ (9) jusqu'ici.

Tous les fidèles, les parents, les personnes ayant des responsabilités ${ }^{15}$ [dans le monastère], les Uk-ñā", les bañ $\bar{a}$, les moines ${ }^{16}$ des provinces voisines ou lointaines, épanouis par leur (10) foi ${ }^{17}$, ayant entendu que j'avais décidé de construire ce sanctuaire, sont venus ensemble, et ils ont réuni des subsides qu'ils [m']ont offerts afin d'acheter (11) ces piliers, pour une somme totale de 20 lingots ${ }^{18} \mathrm{~d}$ 'argent.

Pour construire ce sanctuaire, (12) seuls [ont travaillé] des religieux, tous anciens élèves [du Braḥ Gun Braḥ Ariyasaddhā et du Brạ̣ Gụ Ariyaghosa]. Personne n'a été embauché. Alors qu'on allait monter [la charpente] ${ }^{19}$, le vénérable (13) Ariyasaddhā est tombé gravement malade et il est mort ${ }^{20}$.

(14) Le vénérable Ariyaghosa demeurait seul vivant. [En qualité de] chef du monastère, je suis allé (15) l'inviter à venir bénir ${ }^{21}{ }^{1}$ 'ensemble de nos travaux. Comme maîtres de cérémonie, j'ai sollicité les deux [sic] ${ }^{22} g^{2} \bar{u}^{23}$ (16) ayant pour nom maître An, maître Kèr et maître Ang, qui sont habitués ${ }^{24}$ (17) à préparer les offrandes ${ }^{25}$ pour proclamer l'invitation faite aux divinités afin qu'elles soient bienveillantes ${ }^{26}$. Le moment propice ${ }^{27}$ où l'on a dressé ce sanctuaire (18) fut le lundi ${ }^{28}, 1^{\text {er }}$ jour de la lune décroissante, du mois de

13. Mot à mot: lör $(<l \ddot{o})$ «sur» + khluon è் « soi-même».

14. Mot à mot: nāmm $(<n \bar{a} m)$ « diriger», éventuellement «transporter par flottage» + mak «venir», tal «atteindre».

15. L'expression anak muk anak kā (<anak mukh anak kār), mot à mot «ceux qui ont un visage, ceux qui ont une tâche», désigne les personnes qui ont des responsabilités au sein d'une communauté; en l'occurrence, les laïcs faisant office de «maîtres de cérémonies dans le monastère» (ācāry $v a t t$ ), hommes généralement âgés, attachés au monastère et préposés à l'organisation des différents rituels au cours desquels ils donnent le répons aux religieux, voire au cours desquels ils ont un véritable rôle d'officiant, comme «l'ācāry yogī» pour les rites funèbres (cf. infra, face II, ligne 8).

16. brah, pour brah sangh

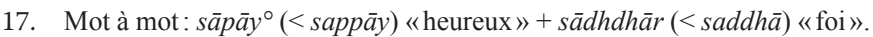

18. «ṇèn», ancienne unité monétaire khmère consistant en une barre d'argent de 385,85 $\mathrm{g}$ (Headley 1977, I: 309).

19. Les piliers et les éléments de la charpente, après avoir été mesurés et ajustés, sont montés en une seule fois. En effet, ce type d'édifice est, au Cambodge, construit sans étais ni échafaudage (comme il en va encore pour la plupart des maisons de bois dans les campagnes).

20. Mot à mot: as $\left(\left\langle a s^{\prime}\right)\right.$ «la totalité» + brahjun $(<$ brah junm $)$ «âge» [pour un religieux] + dov $(<$ dau $)$ «aller».

21. Mot à mot: mak «venir» $+j \bar{a}$ «être» + maingal «bénédiction» + ddham (< dham $)$ "grand».

22. Le texte stipule bien «deux» $\left(b i y^{\circ}<b \bar{i} r\right)$ mais donne trois noms. Il s'agit d'une erreur manifeste du lapicide.

23. Le mot grū (< sk. guru) désigne un «maître» qui enseigne un savoir initiatique, quelle que soit la discipline, dans le cadre personnel de la relation d'un maître à son disciple. Le mot ācāry (sk. $\bar{a} c \bar{a} r y a$ ) se rapporte au «maître» qui dispense un enseignement socialement ouvert. Dans l'occurrence les grū étant également acāry, on se dispense de traduire les deux termes de la même façon.

24. ammrun் (< bamrun்) «être disposé», «être entraîné» ou «être de la réserve» [pour un soldat].

25. $\quad b l \bar{\imath} y{ }^{\circ} k \bar{a} r(<$ balīkār $=$ balikār $=$ blikā $r)$ "préparer des offrandes».

26. Mot à mot: mak «venir» + jā «être» + pațam « en premier lieu» $+k \bar{a}(\langle k \bar{a} r)$ «faire» $+r k$ «être plaisant».

27. bìlā $(<$ belā $) \ll$ instant», «moment».

28. Mot à mot: $\operatorname{nov}(<n \bar{u} v)$ 《lors» + kāl «à l'instant»+ paccapan (<paccuppann) «présent» + thriaiy $^{\circ}$ ccān $(<$ thriai cand $) \ll$ lundi». 
phalgun, de l'année du Cheval, $6^{\mathrm{e}}$ de la décade, pour que, à son achèvement, ce lieu demeure ${ }^{29}$ (19) dans la religion bouddhique indéfiniment.

L'érection de ce sanctuaire ayant [commencé] l'année du Cheval, le travail a été poursuivi (20) jusqu'à l'année du Lièvre. On a [alors] employé des ouvriers pour faire la dorure des poutres ${ }^{30}$ et des piliers au pochoir ${ }^{31}$; ils ont achevé leur travail l'année du Dragon, 6 de la décade, (21) l'an 2447 de l'ère bouddhique.

Quant aux fruits des avantages obtenus pour avoir édifié ce sanctuaire, (22) je requiers de faire l'hommage de ces mérites royaux ${ }^{32}$ qui se reportent sur les vertus de mes deux maîtres, sur les vertus (23) des parents, sur les vertus du roi, sur les grands-parents, sur la parentèle ${ }^{33}$ sur 7 générations ${ }^{34}$. [Ce sanctuaire] étant complètement achevé, (24) qu'il demeure dans la religion de l'auguste vertu dont l'auguste nom est l'Auguste Samanagotam, le maître rayonnant ${ }^{35}$ ! Qu'il demeure (25) jusqu'à l'auguste enseignement de l'auguste [buddha] Seametrey (si-Āry metrīy) ${ }^{36}$ !

Moi, qui suis le chef de ce monastère, j'ai composé ce texte (26) en espérant qu'il demeurera ${ }^{37}$ pour tous les fidèles ${ }^{38}$, autant qu'ils sont, qui, d'un cœur pieux, ont été spontanés ${ }^{39}$; en souvenir (27) des vertus de tous ceux qui m'ont fait don de subsides, modestes ou considérables, [et] qui ont ensemble (28) contribué à l'édification de ce sanctuaire.

Maintenant, la construction de ce sanctuaire étant achevée, alors je requiers d'en obtenir tous les mérites (29) pour tous ceux qui se sont unis sur les Voies [supramondaines] qui portent les fruits [supramondains] ${ }^{40}$,

29. Mot à mot: jā «être» + maígal «lieu béni » + tamkal' «placer», «conserver» $+d u k$ «conserver».

30. Le terme phtèr désigne «la partie d'une maison khmère qui joint le toit aux murs» (Headley 1977, I: 617). Dans le cas précis du vatt Mahā Lābh, il s'agit des poutres de la charpente qui sont, effectivement recouvertes de laque noire ornée de motifs au pochoir dorés (Nafilyan 1997: 13, photo 13).

31. Mot à mot: $g \bar{u} v(<g \bar{u} r)$ «dessiner» + phtèr «poutre» + pit $\left(\langle\right.$ pid $)$ «appliquer», $m \bar{a} s^{\circ}(m \bar{a} s)$ «or», sasar «pilier».

32. Les «mérites» (kusal[a]) sont qualifiés de «royaux» (rājā-) par anticipation sur la suite de la phrase car au nombre des dédicataires figure Sa Majesté le roi.

33. $\tilde{n} \bar{a} t-t ̦ i k \bar{a}<\tilde{n} a \overline{t a k} a$ «parentèle».

34. La dédicace des mérites est faite selon une formule stéréotypée: il s'agit ici des parents, des grands-parents et de la parentèle sur sept générations, de tous ceux qui ont participé à la construction du sanctuaire.

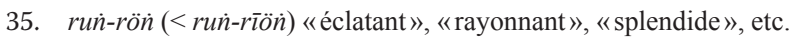

36. Le nom du buddha $s i-\bar{a} r / s ə-a /$ - buddha du futur, cinquième et dernier buddha du présent éon (pā. bhaddakappa, sk. bhadrakalpa) - est la forme abrégée de la désignation khmère usuelle si-Āry metrīy /sə-a métrey/, résultant d'une influence d'une prononciation thaïe - th. /si/ > kh. /sə/ - de la graphie sanskrite Śrī Ariya Maitreya. Le Dictionnaire cambodgien de l'Institut bouddhique ne propose, de façon symptomatique, que la graphie pāli Ariya Metteyya (Chuon Nat 1967: 814).

37. Mot à mot: $j \bar{a}$ «être» + samnnūm « vœu»+duk « conserver».

38. Le terme ñom est, au sens étroit, l'appellatif utilisé par un religieux lorsqu'il s'adresse à ses parents ou, plus largement, lorsqu'il s'adresse à n'importe quel laïc.

39. Mot à mot: tèl «qui » + mān «avoir» + cit (<citt) «cœur», « esprit» + sāddhā (<saddha)

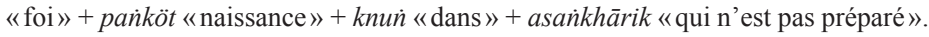

40. Mot à mot: măg (<magg) «voie» + phal «fruit, qui porte des fruits». Ceux qui suivent les quatre «voies supramondaines» (lokuttara-magga) et possèdent les quatre «fruits supramondains » (lokuttara-phala) sont les «nobles» (i.e. «saints») (ariya puggala). 
pour les fidèles laïques, hommes et femmes, jusqu'à ce que [l'auguste buddha] Seametrey prêche sa doctrine. Alors, je requiers pour eux qu'ils (30) atteignent les voies qui portent les fruits qui conduisent au nibbāna, lieu de l'absolue félicité.

\section{[Face II]}

(1) Proclamation ${ }^{41}$.

(2-3) Sa sainteté le suprême patriarche, qui est le chef de tous les religieux (4) du royaume du Cambodge ${ }^{42}$, (5) conducteur suprême des affaires monastiques des vénérables de tous rangs, (6) dans les 4 ensembles de religieux appartenant à la hiérarchie monastique, donne acte ${ }^{43}$, au vatt Mahā Lābh dans la province de Tpūn Ghmum, (7) afin que cela parvienne à la connaissance de tous ${ }^{44}$, que, s'agissant de ce monastère, [le chef du monastère] - le Brạ̣ Sāsanānuvañs - (8) ainsi que tous maîtres [laïcs] qui [y] exercent une responsabilitée ${ }^{45}$, l'ensemble des [fidèles] hommes et femmes, ayant achevé de l'édifier, (9) en ont fait l'hommage à Sa Majesté le roi car (10) Elle avait ordonné Braḥ Sāsanānuvañs, qui est chef provincial des religieux, ainsi qu'au Brạ̣ Sāsanalikhit, son adjoint, (11) qu'ils convainquent tous maîtres [laïcs] ayant une responsabilité, l'ensemble des [fidèles] hommes et femmes, [habitant] dans le voisinage ou plus loin de se décider à poser les bornes (12) rituelles.

Sa Majesté le roi a été invitée, par le Braḥ Dhammalikhit, qui est le chef de la province (13) ainsi que par ses vénérables assistants ${ }^{46}$, le Brạ Pālāât' Saṅghavañs, le Brạ̣ Pālāat Sañghavijjā, le Braḥ Ariyācāry et le Brạ̣ Dhammapati, à apposer sa royale signature ${ }^{47}$.

(14) Sa Majesté le roi du Cambodge est convenue ${ }^{48}$ qu'il soit procédé aux rites qui consistent à poser des bornes rituelles. [Cette cérémonie] a

41. L'expression $r a \bar{j} j$-prakās, qui signifie mot à mot «proclamation royale», est utilisée ici pour désigner une proclamation issue par le «roi des bonzes» (sangharāj) et non par le souverain du Cambodge. L'utilisation du «vocabulaire royal» (rājasabd) pour et par les grands religieux - comme, par exemple, le pronom $d r a \dot{n}^{\prime}$ («il») (ligne 6) - est largement tombée en désuétude au $\mathrm{XX}^{\mathrm{e}}$ siècle.

42. La formule qui désigne le Cambodge, ici encore, emphatique et conventionnelle, est, mot à mot: «la cité de l'état du Kambūjā» (krun் kambūjāradhdhā < krun் kambujarațh), «grande cité d'Indapatta» (mahā indapatt nagar), «heureuse extrêmement» (pūrīraṃm param pabity $<$ purīramy param pabitr).

43. Mot à mot: $t \bar{a} \dot{n}^{\circ}(<t \bar{a} \dot{n})$ «témoigner»+duk «placer», « conserver».

44. ānāprajājun (< ānāprajājan) désigne tous les habitants d'un royaume (ānācakr).

45. anak mak anak kār (< anak mukh anak kār), cf. supra, face I, ligne 9.

46. Le texte reprend le titre de $p \bar{a} l \bar{a} t^{\prime}$ « assistant [d'un megan] » pour qualifier chacun des quatre religieux désignés ici. Ces titres sont, aujourd'hui, tombés en désuétude.

47. Mot à mot: lậy «signer», brah hasaḷkkhā (< brah hastalekhā) «signature» (vocabulaire royal). La nature du document signé par le roi n'est pas précisée dans l'inscription, mais son contenu est décrit dans les lignes qui suivent (cf. infra, ligne 14).

48. L'expression mak dhvö «venir faire» est curieuse, sans doute fautive. En tout état de cause, elle ne signifie pas que le roi est venu lui-même parce que le verbe utilisé, pour signaler un déplacement royal, aurait été yāin mak. 
(15) commencé le vendredi, $15^{\mathrm{e}}$ jour de la lune croissante du $4^{\mathrm{e}}$ mois. L'enfouissement des racines ${ }^{49}$ a eu lieu le lundi, $3^{\mathrm{e}}$ jour de la lune décroissante $\mathrm{du} 4^{\mathrm{e}}$ mois de l'année du Serpent, $7^{\mathrm{e}}$ de la décade, à (16) la $1^{\text {re }}$ heure khmère, correspondant à la $7^{\mathrm{e}}$ heure française. Cela étant accompli, on a invité cent augustes moines à prendre part à un sang $\bar{a} y a n \bar{a}^{50},(17)$ l'après-midi pour clore la cérémonie ${ }^{51}$. Sa Sainteté ${ }^{52}$ en a pris acte, le mercredi, $5^{\mathrm{e}}$ jour de la lune décroissante du $4^{\mathrm{e}}$ mois de l'année 1267 de la petite ère, (18) l'année du Serpent, $7^{\mathrm{e}} \mathrm{de}$ la décade, équivalent au quatorze mars de l'année occidentale 1906.

(19) Sa Majesté a eu la bonté de faire, de tout son cœur, les dons [nécessaires] aux religieux pour qu'ils procèdent à la pose des bornes.

(20) Daigne Sa Majesté, le maître des vies sur les têtes ${ }^{53}$, accorder la permission $^{54}$ royale de saluer Sa (21) Majesté le roi, maître des vies sur les têtes; daigne l'Auguste Poussière des Augustes Pieds ${ }^{55}$ être informée (22) que le Brah Sāsanalikhit, adjoint [du chef provincial des religieux $]^{56}$, chef du monastère de Ṭaṃnāk’ Brīn, province de Tpūn Ghmuṃ, (23) a requis d'adresser des vœux pour acquérir le terrain ${ }^{57}$ afin de poser les bornes rituelles

49. Le rituel désigné ici par l'expression $k \bar{a} t$ rös $\left(<k \bar{a} t^{\prime} r r_{s}\right)$ consiste à sectionner $\left(k \bar{a} t^{\prime}\right)$ simultanément d'un coup, les liens par lesquels les neuf racines $(r s)$ de $s \bar{m} \bar{a}$ sont suspendues au-dessus de fosses au fond desquelles elles tombent et demeurent enfouies.

50. Le terme technique samghāyanār (< sañgāyanā «récitation en commun») désigne, en principe, des réunions solennelles, étendues sur plusieurs jours, organisées à l'instar des premiers Conciles bouddhiques, au cours desquelles de grands religieux lettrés pratiquent des «récitations de vérification» du texte des Trois Corbeilles afin d'éliminer les inexactitudes qui ont pu s'introduire au cours de sa transmission.

Dans la langue khmère, ce terme désigne, soit certains textes en langue vernaculaire, soit le rituel au cours duquel sont utilisés ces textes, lus par deux, trois ou cinq religieux - selon qu'il s'agit du sañgāyanā grè bīr, -grè pi, -grè prām c'est-à-dire la «vérification [du vināya] à deux, trois ou cinq chaires ») - qui s'interrogent et se répondent l'un l'autre à propos de questions de discipline monastique, chacun d'eux incarnant, à cette occasion, l'un des grands acteurs du Premier Concile bouddhique. L'hypothèse que le mot sangāyanā puisse, dans cette occurrence, signifier simplement «chanter ensemble», et donc que la phrase «(...) jā srec hoÿ nimmant brah saìgh 100 ang samghāyanār velā rasiel jā höy ${ }^{\circ}$ puny» puisse signifier « (...) cela étant accompli, cent religieux ont été invités à chanter ensemble, pendant l'après-midi, ce qui a constitué la clôture de la cérémonie», a été rejetée catégoriquement par M. Kun Sopheap, collaborateur scientifique principal de l'EFEO-FEMC.

51. L'expression jā höy puny (<jā höy puny) est la forme syncopée de jā kicc paṅhöy puny, ou jā pan்höh puny «en guise de clôture de la cérémonie», formule qui appartient, en tout état de cause, à la langue parlée.

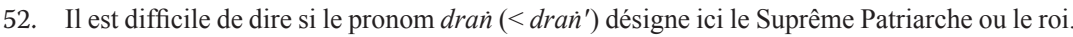

53. Mot à mot: brah țèjjah (< braḥ tejah) «auguste puissance», țamkal (< tamkal') "placée», $l \ddot{o}$ «sur», thpǜi $(<t p \bar{u} \dot{n})$ «les têtes».

54. Mot à mot: sūm «requérir», brah rājadān «l'auguste don [i.e. la permission] royal», krāp pañgamm (< krāp pañgam) «s'incliner respectueusement», dūl «informer» (vocabulaire royal). Cette formule stéréotypée sert de formule de conclusion pour une adresse au roi (cf. infra, ligne 26).

55. La formule dhüli brah pād «poussière des augustes pieds» est communément attestée au Cambodge depuis l'époque pré-angkorienne, pour désigner le souverain. Elle trouve son origine dans le fait que les sujets prosternés aux pieds du roi, avec l'interdiction de lever les yeux, ne voient effectivement que la poussière soulevée par ses pas. On observe que cette expression d'origine indienne, vexatoire pour les sujets du roi, est la seule formule de l'ancienne titulature royale qui ait traversé les siècles, de l'ère pré-angkorienne jusqu'à l'époque moderne.

56. Cf. supra, ligne 9.

57. Le terme rājadhān̄ $\left(r \bar{a} j-^{\circ}\right.$ « royal» + dhān, «cité») «capitale royale» est utilisé, par métaphore emphatique, pour désigner les terrains qui, par nature, appartiennent tous au roi dans le royaume, sur lesquels sont construits les monastères bouddhiques. 
vatt Mahā Lābh (24) dans la province de Tpūn Ghmuṃ le vendredi, 15e jour de la lune croissante du $4^{\mathrm{e}}$ mois, de cette année du Serpent, $7^{\mathrm{e}}$ de la décade.

Le jour où la pose [des bornes] aura été achevée ${ }^{58},(25)$ il a requis de faire l'hommage [au roi] des mérites acquis ${ }^{59}$ pour ce qu'ils valent: s'ils sont modestes ${ }^{60}$, que Sa Majesté ait la bonté de lui pardonner. Daigne, Sa Majesté, (26) permettre qu'on La salue respectueusement.

Le [mercredi] $13^{\mathrm{e}}$ jour de la lune croissante du $4^{\mathrm{e}}$ mois, de l'année du Serpent, correspondant au 7 (27) mars 1906. [Signé] Uk-ñā Dhamma Udaiy ${ }^{61}$.

\section{Dates et systèmes de datation dans l'inscription K. 1046}

L'inscription K. 1046 fait mention d'un certain nombre de dates correspondant à la gravure de chacune des faces de la stèle, ainsi qu'aux divers événements qu'elles relatent. Pour formuler ces dates les auteurs de ces textes font usage, de façon combinée ou non, de plusieurs systèmes de datation:

a) le millésime de chaque année indiqué dans quatre ères chronologiques différentes: «l'ère bouddhique» (buddhasakarāj), la «grande ère» (mahāsakarāj), la «petite ère» (cullasakarāj), parfois «l'ère chrétienne» (grist-sakarāj);

b) le cycle chronographique duodécimal des animaux: jūt, Rat; chlūv, Bœuf; khāl, Tigre; thoh, Lièvre; ron, Dragon; msā̃n, Serpent; mamī, Cheval; mamè, Chèvre; vak, Singe; rakā, Coq; ca, Chien; kur, Porc (Billard 1963, 403 sqq.);

c) le système décadaire de référencement des années : Ekasăk, première année de la décade; dosăk, deuxième année; trīsăk, troisième année; catvāsăk, quatrième année; pañcasăk, cinquième année; chasăk, sixième année; saptasăk, septième année; ațthasăk, huitième année; nabvsăk, neuvième année; samrịddhisăk, dixième année de la décade.

Au Cambodge, «l'ère bouddhique» (buddhasakarāj), censée débuter le jour du parinibbāna du Buddha, commence en 543 A.D. Elle n'y est guère utilisée avant le $\mathrm{XVI}^{\mathrm{e}}$ siècle.

La «grande ère» (mahāsakarāj), qui commence le 15 mars 78 A.D., n'est autre que l'ère des Śaka, directement importée de l'Inde et servant, à l'exclusion de toute autre, à dater les inscriptions lapidaires khmères et sanskrites du $\mathrm{VI}^{\mathrm{e}} \mathrm{au}$ $\mathrm{XV}^{\mathrm{e}}$ siècle. Elle n'est désignée comme étant la «grande ère» que lorsqu'apparaît l'usage de la "petite ère» (cullasakarāj), au mileu du XIX siècle.

58. L'utilisation, ici, du futur antérieur dans la traduction est nécessitée par la structure narrative temporelle qui semble faire ici, curieusement, un «flash-back», les intentions du religieux initiateur du projet de construction du vatt Mahā Lābh, exposées dans cette fin de texte, étant nécessairement antérieures à la réalisation de ce projet dont la stèle entière donne acte.

59. Les «mérites» (kusala) sont qualifiés ici de «royaux» (räjakusal) du fait qu'ils sont dédiés au roi.

60. Mot à mot: guor bvam guo (< guor bum guor), «digne [ou] indigne», «ayant de la valeur [ou] sans valeur».

61. Uk-ñā Dhamma Uvdaiy ${ }^{\circ}\left(<u k \tilde{n} \bar{a}^{\prime \prime}\right.$ dhamma udăy) : titre de haut fonctionnaire royal. 
La «petite ère» (cullasakarāj $j$ ) commence le lundi, $12^{\mathrm{e}}$ jour de la lune croissante du mois de cetr de l'année du Porc (kur), première de la décade (Ekasăk) de l'année bouddhique 1183, soit le 21 mars 638 A.D. Cette ère, qui semble être originaire du royaume $p y u$ de Srī Kśetra (Vinay 2003 : 67), est utilisée au Cambodge pour la première fois sur les monnaies médailles frappées sous le roi Ang Duong pour commémorer la date officielle de son avènement au trône le mardi, $3^{\mathrm{e}}$ jour de la lune croissante $\mathrm{du} 4^{\mathrm{e}}$ mois de l'année de la Chèvre, $9^{\mathrm{e}}$ de la décade, 1209 cullasakarāj, 1769 mahāsakarāj, 2390 buddhasakarāj, c'est-à-dire le mardi 7 mars 1848 A.D. (ici, l'ère chrétienne correspond à cullasakarā $[638]+1$, puisqu'on est déjà au $4^{\mathrm{e}}$ mois luni-solaire, c'est-à-dire presque à la fin de l'année cambodgienne ${ }^{62}$ ).

Face I, lignes 1 et 2: 2437 buddhasakarāj, 1816 mahāsakarāj, 1256 cullasakarāj $j=1894$ A.D.

Face I, lignes 18-19: «le lundi, $1^{\text {er }}$ jour de la lune décroissante, du mois de phalgun [quatrième mois du calendrier luni-solaire khmer, correspondant généralement à février-mars], de l'année du Cheval (mamī), $6^{\mathrm{e}}$ de la décade [2437 buddhasakarāj]]», c'est-à-dire le lundi 20 février 1894 A.D.

Face I, ligne 20: «l'année du Lièvre (thoh) [ $5^{\mathrm{e}}$ de la décade, 2446 buddhasakarāj] » c'est-à-dire 1903 A.D.

Face I, ligne 20-21 : «l'année du Dragon (ron̈), 6 e de la décade (chasăk), l'an 2447 buddhasakarāj », c'est-à-dire 1904 A.D.

Face II, ligne15 = ligne $24:$ «le vendredi, $15^{\mathrm{e}}$ jour de la lune croissante $\mathrm{du} 4^{\mathrm{e}}$ mois [c'est-à-dire du mois de phalgun de l'année du Serpent, $7^{\mathrm{e}}$ de la décade]», c'est-à-dire le vendredi 9 mars [1906 A.D.].

Ibidem: «le lundi, $3^{\mathrm{e}}$ jour de la lune décroissante du $4^{\mathrm{e}}$ mois de l'année du Serpent, $7^{e}$ de la décade», c'est-à-dire le lundi 12 mars 1906 A.D.

Face II, lignes 17-18: «le mercredi, $5^{\mathrm{e}}$ jour de la lune décroissante du $4^{\mathrm{e}}$ mois de l'année 1267 de la petite ère, (18) l'année du Serpent, $7^{\mathrm{e}}$ de la décade, équivalent au quatorze mars de l'année occidentale 1906».

Face II, lignes 26-27: «le 13e jour de la lune croissante du $4^{\mathrm{e}}$ mois, de l'année du Serpent, correspondant au 7 mars $1906 »$ », c'est-à-dire le mercredi 7 mars 1906.

\section{Événements relatés dans les deux textes formant l'inscription K. 1046}

Les deux textes, inscrits respectivement sur chacune des faces de la stèle, ont été composés par deux auteurs différents. Ils relatent des événements

62. Il existe, dans le corpus des inscriptions du Cambodge, deux occurrences pour l'utilisation de l'ère cullasakarāj antérieures à 1848 A.D. : l'inscription K. 27, datée de 949 cullasakarāj, c'està-dire de 1587 A.D. (Khin 1980 : 125), et l'inscription K. 75 datée de 990 cullasakarāj, c'est-à-dire de 1628 A.D. (Pou 1989: 33). Toutefois, la première de ces deux inscriptions est manifestement un texte khmer adapté du siamois, et la seconde a été composée à la fin du règne de Suryopor par un prince éduqué en Thaïlande. 
connexes mais distincts qui ont eu lieu à des dates différentes. Chacun de ces textes semble avoir été gravé par un lapicide également distinct: la forme des lettres n'est pas la même sur l'une et l'autre face. La souscrite de la lettre $-r$, par exemple, est arrondie sur la face I, tandis qu'elle se termine systématiquement par un crochet ornemental sur la face II.

Le texte de la face I (K. 1046 a), composé en 1904 A.D. par le chef de monastère du vatt Mahā Lābh, le vénérable Ind-Jot, relate les circonstances qui avaient présidé à la construction du vihāra, commencée en 1894 A.D., dix ans donc avant la gravure de l'inscription.

Le texte de la face II (K. 1046 b) a été rédigé deux ans plus tard, en 1906 A.D. par le «gouverneur» (cauhvāy sruk) de la province, dont le «nomtitre» (jhmoh-īnar) Uk-ñā Dhamma-Udaiy (cf. face II, ligne 27) est calqué sur celui de l'ancien «roi feudataire» (stec trāañ') du ți de Tpūn Ghmum, l'Uk-ñā Dhammātejo. Le commanditaire de cette inscription est désigné dès la première ligne: il s'agit bien du suprême patriarche dont le nom personnel n'apparaît toutefois pas.

Ce second texte relate la cérémonie solennelle de pose des «bornes rituelles» $(\operatorname{sèm} \bar{a}<\operatorname{sim} \bar{a})$ organisée sous le haut patronage royal. Les «bornes rituelles » d'un monastère, implantées aux huit points cardinaux et intercardinaux ainsi qu'au centre du sanctuaire devant la statue principale du Buddha, sont formés de deux éléments : la "racine" (rss sìmā), constituée d'un gros bloc de pierre arrondi enfoui dans le sol et, sauf pour le simma du centre du sanctuaire, d'une "feuille" (slik sīmā), formée d'une stèle plate dont le sommet dessine généralement une accolade horizontale, souvent ornée de motifs. La cérémonie de pose des «bornes rituelles» (sìmā) marque la véritable fondation d'un monastère en ce sens que, à compter de la date de ce rituel solennel, la communauté des religieux qui l'habite ne dépend plus organiquement d'un autre vatt et peut accomplir sur place de façon valide les «actes de la vie communautaire» (samghakamma), comme la «prise d'habit monastique» (bìdhi pampuos), la récitation de la «règle» (pațimokkha), etc.

Le terme pañcuh (formé de $p \bar{a} \tilde{n}-\{$ modificateur actanciel $\}+c u h$ «descendre») désigne ici l'acte qui consiste à enfouir dans la terre les «racines de $\operatorname{sim} \bar{a} \gg(r s s \bar{l} m \bar{a})$ et, par extension, la cérémonie entière au cours de laquelle ces objets sont simultanément enfouis dans le sol (cf. infra, ligne 24).

Le terme technique samghakamm (< sainghakamm) (cf. face II, ligne 14) désigne strictement les «actes monastiques» qui excluent la participation active de laïcs. S'il est constant que la pose des bornes rituelles dans les monastères donne lieu à de grands rituels au cours desquels la foule des fidèles laïcs, et notamment les «maîtres de cérémonies de la pagode» (ācāry $v a t t$ ) ont un rôle prépondérant, ces rituels sont précédés du rite fondamental visant à délimiter et à dé-sacraliser par précaution le sol sur lequel va être consacrée la clôture rituelle du sanctuaire - de peur d'empiéter sur un terrain consacré autrefois, voire très anciennement à l'époque d'un buddha du passé - puis à le re-consacrer. Ces rites ne peuvent effectivement être accomplis que par des bhikkhu qui s'isolent en refermant sur eux les portes du sanctuaire (vihāra), hors de la présence et même hors de la vue de tout laïc. 


\section{Éléments de toponymie religieuse}

Outre le vatt Mahā Lābh, l'inscription K. 1046 fait mention de plusieurs autres monastères: l'un est immédiatement voisin du monastère, les deux autres un peu plus distants.

Face I, ligne 5 : vatt Kaṇtal (<kantāl $)$ «monastère central»). Les monastères du Cambodge portent généralement un «nom officiel» (jhmoh phlūv $k \bar{a} r$ ) formé de termes $p \bar{a} l i$, que les habitants eux-mêmes ne connaissent pas toujours et qui, au demeurant, peut changer au fil du temps. À côté de ce nom officiel, ils ont un «nom d'usage» (jhmoh hau) vernaculaire qui est à la fois mieux connu et plus stable dans le temps. Vatt Kantal est, en l'occurrence, le nom usuel de deux monastères du district de Koḥ Sūdin: le vatt Bodhi Riddhī, dit vatt Koḥ Kaṇtāl et le vatt Visuddhīvañs, dit encore vatt Koḥ Kaṇtāl. Faute d'aucun indice spécifique, il est impossible d'identifier précisément lequel de ces deux monastères est celui de résidence des deux religieux mentionnés dans le texte.

Face II, ligne 22: vatt Ṭạ̣ṇāk' Brīn (<țaṇnāk' brīin) (/damnak pring/) «monastère du parc aux “jambosiers" (Syzygium jambos)». Ce vatt, situé dans le village de Ṭạ̣nāk’ Brīn est voisin du vatt Mahā Lābh, dans l'actuel «canton» (ghum) de Mahā Lābh du «district» (sruk) de Koḥ Sūdin. Il convient de noter à son propos que, si le vatt Mahā Lābh a miraculeusement réchappé de la guerre de 1970-1975 et de la fureur anti-religieuse des Khmers Rouges qui l'avaient transformé en hôpital, le vatt Ṭaṃnāk' Brīn, en revanche, a été anéanti lors des épouvantables bombardements américains du 5 juillet 1970 qui firent périr en quelques minutes tous les religieux présents et toute la population civile qui avait cru pouvoir y trouver protection.

\section{Éléments de titulature religieuse et civile}

L'inscription K.1046 contient un certain nombre de «noms-grades» (jhmoḥ $\dot{n} \bar{a} r)$ de religieux correspondant à divers niveaux de la «hiréarchie dans la communauté [monastique]» (rāja $\bar{a} g a n a:$ ), ou bien de «noms-fonctions » (tuo $n \bar{a} d \bar{l})$ désignant une fonction particulière exercée au cours d'un rituel spécifique ou bien dans l'organisation locale du clergé bouddhique.

Face I, ligne 3 : l'un des religieux est désigné, dans une occurrence, à la fois par son nom-fonction et son «nom propre d'ordination» (nām pañnatti): il s'agit du brah cauv ațthikā (< brah cau adhikār "vénérable chef de l'administration ») du vatt Mahā Lābh, dont le nom propre de religieux, reçu le jour de son ordination, est Ind-joti («splendeur d'Indra»). Le titre religieux d'Upajjhā (< Uppajjhāya) qui lui est donné dans l'inscription, désigne techniquement le "précepteur religieux» qui, avec le «maître d'ordination » (kammavācācārya), préside la cérémonie d'ordination d'un nouveau religieux. Ce titre, qui est normalement porté de façon circonstancielle, c'est-à-dire à l'occasion d'une cérémonie particulière au cours de laquelle tel vénérable est invité à assumer le rôle de «précepteur religieux». Dès lors que l'événement relaté dans la présente inscription n'a rien à 
voir avec un rituel d'ordination, il faut comprendre que le titre d'Upajjhā désigne ici un personnage exerçant cette fonction de façon statutaire dans «l'arrondissement» (sruk) où il réside.

Face I, ligne 4: brah gun braḥ ariyyasatthā $(<$ braḥ gun braḥ ariyasaddha $\bar{a}$, mot à mot pa . ariya «noble» + saddha «foi») est un titre porté par un religieux de rang moyen, responsable de la discipline monastique dans un district donné. Dans les années 1960-1970, par exemple, il était porté par le «chef des religieux» (anugun sruk) du district de Thban (/thpong/) dans la province de Kompong Speu (Chuon Nat, 1976: 46).

Face I, ligne 4 : brah gun brah ariyyaghosār $(<$ brah gun ariyaghosa, mot à mot $p \bar{a}$. ariya «noble»+ghosa «formule») est tombé en désuétude.

Face I, ligne 9: Aokña ( $<$ Uñ̄a $\left.\bar{a}^{\prime}\right)$ est un titre civil réservé, jusqu'au milieu $\mathrm{du} \mathrm{Xx}^{\mathrm{e}}$ siècle, à des fonctionnaires de très haut rang, ministres ou gouverneurs de province. Depuis la restauration du royaume en 1992, il est principalement utilisé comme signe de distinction pour des personnes éminentes dans la vie économique du pays, notamment pour des magnats dont la fortune, souvent rapide et récente, a été favorisée par un pouvoir assuré, en retour, de leur soutien inconditionnel.

bañā, titre dérivé du thaï brah-ñā /paya/ ou du Lao baḥña /paya/ (Chuon Nath 1967: 725b; Headley 1977: 629). Ce titre princier à l'origine - qui entre notamment dans la composition du nom du roi baña-ya"t, le Ponhea yat des Chroniques - ne désigne plus au fil des siècles que des fonctionnaires de rang subalterne, subordonnés dans l'administration territoriale aux gouverneurs des provinces.

Face II, lignes 2 et 3 : la traduction conventionnelle par «suprême patriarche» résume ici les éléments d'une titulature religieuse pléthorique et redondante, également conventionnelle, qui cherche davantage à produire un effet d'emphase qu'à définir de façon analytique la fonction du personnage ainsi désigné: «seigneur» (sammtec< < samtec), «auguste grand roi des religieux» (brah mahā samgharājā < brah mahā sañgharāj), « commandeur du clergé» (gaṇädhiptī < gaṇa + ādhipatī), «sacré suprême chef des bonzes» (sirī paramm pabits saṃhanāya ${ }^{\circ} k<$ srī param pabitr sañghanāyak), «merveille dans le monde» (tilukalokā < tilakalokā), «doué d'une morale sublime» (carīya țamm < cariyā uttam), «supérieur à tous» (paramedh $<$ paramesdh), «le plus grand parmi les religieux» (sainghajetțha), «grand roi des religieux extrêmement suprême» (mahā saṃharāja varoțạmm param pabity < mahā saingharāj varottam param pabitr). À la date de cette inscription, le suprême patriarche du Cambodge est, comme on l'a vu, le vénérable Nil Dīen (1823-[1857]-1913).

Face II, ligne 6: le terme rājāganah (< rājāgana:), mot à mot «groupe royal», désigne dans l'usage les vénérables moines appartenant, autour du Suprême Patriarche, à la haute hiérarchie du sanigha khmer et, par extension, l'ensemble des titres portés par ces vénérables.

L'expression rājāgaṇah dāmn் 4 saṃrāp (< rājāgaṇa: dāṃn 4 samrāp') fait ici référence à la division traditionnelle du sarigha khmer qui, jusqu'à 
la fin $\mathrm{du} \mathrm{XIX}^{\mathrm{e}}$ siècle, reflétait la division plus ou moins réelle du pays en quatre «ensembles [de provinces]» (samrāp $\left.{ }^{\prime}\right)$, connus généralement en français comme les quatre «apanages»: celui du roi, comptant trente-neuf provinces; celui du «roi ayant abdiqué» (mahā upayorāj), comptant cinq provinces; celui du «second roi» (mahā uparāj), comptant cinq provinces; celui de la reine mère (vararājinī), comptant quatre provinces (Sam Yang 1990 : 86). Les religieux du premier groupe, désignés par le roi, constituaient le brah rājagaṇa: samrāp' Ek; ceux du second groupe, désignés par l'upayorāj constituaient le brah rājagana: samrāpl do; ceux du troisième groupe, désignés par l'uparāj constituaient le brạ rājagana: samrāp' trī, et ceux du quatrième groupe, désignés par la reine mère, constituaient le brạ rājagaṇa: samrāp' catvā (Damrong 1935 : 38).

Les prébendes et les rentes attachées aux «apanages» traditionnels ont été supprimés par le Protectorat en janvier 1877 (Lamant 1985 : 332). En outre, en 1906, à la date de l'inscription, il n'y avait pas de mahā upayorāj au Cambodge et la position même de mahā uparāj avait disparu avec l'avènement au trône du dernier titulaire, le roi Sisowath lui-même, qui n'avait pas désigné de nouvel uparāj. L'évocation, en 1906, des quatre samrāp religieux ne traduit donc que la permanence purement formelle de l'ancienne division du rājāgaṇah.

Face II, ligne 9: la formule dran brah kūṇa bōy ${ }^{\circ}$ sèsțh jā mcāh $(<$ dran்' brạ karuṇa bises jā mcās' [jīvit tamkal' lö tpǜi]): «auguste miséricorde extraordinaire qui est le maître [des vies qui est placé sur les têtes]» est usuelle pour désigner le roi. Le pronom initial drañ n'a pas ici de justification grammaticale. Dans l'occurrence, le roi du Cambodge sous le règne duquel ont été composées les inscriptions figurant sur la stèle, et sous le patronage duquel a lieu la cérémonie décrite dans l'inscription de la face II, est le roi Sisowath (1840- [r.1904]-1927).

Face II, ligne 7: le titre de brah sāsanānuvañs dhammacarīyā mahā gana: («celui qui suit la religion, maître de doctrine de la grande section») était le titre complet porté par le chef du monastère Mahā Lābh, lequel exerçait, en outre, la fonction de chef des religieux de la province (cf. face I, ligne 3). Ce titre n'est plus en usage.

Face II, ligne 10: le titre de brạ sāsanaḥ likkhit (< braḥ sāsanalikhit) ( «celui qui retranscrit la religion») était le titre porté par «l'assistant suppléant» (pālāat' rà̉ rājkār) du megaṇ.

Face II, ligne 12: le titre de brah dhammalikkhit (< brah dhammalikhit) («celui qui retranscrit le dhamma») est le plus élevé de toute la «hiérarchie religieuse » (rājagana:). Il apparaît immédiatement après celui de Suprême Patriarche. Le titre de kuṃnān (< kaṃnānn') désigne, dans l'administration civile, le chef de cent maisons. Sachant que le brah sāsanānuvañs est déjà désigné comme étant le chef provincial des religieux (ligne 10), il n'est pas aisé de se figurer à quelle fonction particulière correspondait le titre de kamnnạn' khett pour un personnage aussi considérable dans le royaume que le brah dhammalikhit. 
Face II, ligne 13: les quatre titres religieux que sont brah samghavañs (<brah sanghavains : «celui qui appartient à la communauté [monastique]»); brah samvijār (<brah sanghhavijjä : «celui qui enseigne à la communauté»); brah ariyaca $\bar{a}$ (brah ariyaccā); brah dhammaputī («celui qui appartient au dhamma») ne font plus partie de la titulature actuelle du rājagana:

Chacun de ces quatre religieux est qualifié ici de $p \bar{a} l \grave{a} t$. Le terme $p \bar{a} l \bar{a} t$ $\left(<p \bar{a} l \bar{a} t^{\prime}\right)$ est issu, par dérivation, de la racine khmère plas «changer», empruntée par le thaï puis restituée après transformation de la finale /-s/ en dentale/t/ conformément à la phonétique siamoise (Pou, 1989: 94). Il désigne donc «celui qui remplace», «le substitut». Dans le vocabulaire religieux moderne, le pālāt' désigne le plus souvent l'assistant d'un megan («responsable de la communauté monastique») dans un district ou dans une province.

\section{Bibliographie}

Aymonier, Étienne

1900 Le Cambodge, I. Le royaume actuel, Paris, Ernest Leroux.

BILLARD, Roger

1963 «Les cycles chronographiques chinois dans les inscriptions Thaïes », Bulletin de l'École française d'Extrême-Orient 51 (2), p. 403-431.

CHUON Nath [Samdec braḥ Mahāsumedhādhipatī Chuon Nat Jotaññāṇo]

1967 Vacanānukram khmèr [Dictionnaire Cambodgien], Phnom Penh, Institut Bouddhique.

1976 «The Governing of the Buddhist Order in Cambodia», Visakha Puja, p. 40-47.

DAMrong (Prince) [en thaï]

1935 (2438) Nirās Nagar Văt [Nostalgie d'Angkor Vat], Bangkok, [livre commémoratif en l'honneur de l'anniversaire de Prah Sophana Aksonkit].

De FÉo, Agnès

2005 «Le royaume bouddhique face au renouveau islamique», Les cahiers de l'Orient 78, p. 99-114.

GrosLIER, George

1931 Eaux et lumières. Journal de route sur le Mékong cambodgien, Paris, Société d'éditions géographiques, maritimes et coloniales. [Rééd. : 2008, Paris, La Bibliothèque (L'écrivain voyageur)].

Headley, Robert K. Jr.

1977 Cambodian-English Dictionary, Washington, D.C., The Catholic University of America Press, 2 vol.

KHIN Sok

1980 «L'inscription de vatta Romlok K $27 »$, Bulletin de l'École française d'Extrême-Orient 67, p. 125-131+ 1 planche. 
LAMANT, Pierre Lucien

1985 «Survivre pour renaître: le protectorat français sur le Cambodge (1863-1953)», Indochine. Alerte à l'Histoire, Paris, Académie des sciences d'outre-mer, Institut de l'Asie du Sud-Est, p. 329-340.

Le Bonheur, Albert

1989 Angkor. Temples en péril, Paris, Hersher.

LECLÈRE, Adhémard

1894 Recherches sur le droit public des Cambodgiens, Paris, Augustin Challamel.

Mak Pheun

1995 Histoire du Cambodge, de la fin du XVI ${ }^{e}$ siècle au début du XVIII ${ }^{e}$, Paris, École française d'Extrême-Orient (Monographie, 176).

MiNISTÈRE DE L'INFORMATION

1962 Cambodge, Phnom Penh, Ministère de l'Information du Gouvernement royal du Cambodge.

NAFILYAn, Jacqueline \& Guy

1997 Peintures murales des monastères bouddhiques au Cambodge, Paris, Maisonneuve \& Larose/Éditions de l'UNESCO.

Pou, Saveros

1989 Nouvelles inscriptions du Cambodge, Paris, École française d'Extrême-Orient (Collection de textes et documents sur l'Indochine, 17).

VINAY Pongsiphien [en thaï]

2003 (2546) văn vār kāl velā lee nānā śăkarāj [Les jours, les heures et les différentes ères], Bangkok, krom vijarak, krasuong seksathikan [Service des études, ministère de la Recherche].

SAM YANG

1990 «Buddhism in Cambodia, 1795-1954», A Thesis Presented to the Faculty of the Graduate School of Cornell University in Partial Fulfillment of the Requirements for the Degree of Master of Arts [non publié]. 